\title{
A Role for Synaptotagmin VII-Regulated Exocytosis of Lysosomes in Neurite Outgrowth from Primary Sympathetic Neurons
}

\author{
Rosa M. E. Arantes ${ }^{1}$ and Norma W. Andrews ${ }^{1,2}$ \\ ${ }^{1}$ Section of Microbial Pathogenesis and ${ }^{2}$ Department of Cell Biology, Yale University School of Medicine, New Haven, Connecticut 06536
}

\begin{abstract}
Neurite outgrowth is mediated by the exocytosis of intracellular vesicles at the tips of elongating neuronal processes. The lysosomal vesicle-associated soluble $N$-ethylmaleimide-sensitive factor attachment protein receptor tetanus neurotoxin insensitive vesicleassociated membrane protein (TI-VAMP)/VAMP7 was previously implicated in membrane fusion events mediating neurite outgrowth, but the participation of lysosomes in this exocytic process has remained unclear. Here, we show that VAMP7 and the lysosomal glycoprotein Lamp1 extensively colocalize in vesicles present throughout the soma and neurite outgrowths of primary sympathetic neurons. Synaptotagmin VII (Syt VII), a $\mathrm{Ca}^{2+}$-sensing synaptotagmin isoform previously shown to interact with VAMP7 during lysosomal exocytosis in fibroblasts, was detected on a subset of these lysosomal glycoprotein 1 (Lamp1)/VAMP7-positive neuronal vesicles. Ionophore-stimulated exocytosis triggered exposure of the luminal domains of both Lamp1 and Syt VII at overlapping sites on the neuronal surface, indicating that the Syt VII-containing lysosomal compartments fuse with the plasma membrane in response to $\left[\mathrm{Ca}^{2+}\right]_{\mathrm{i}}$ elevation. To determine whether Syt VII was required for the exocytic events mediating neurite extension, we followed the development of superior cervical ganglion neurons explanted from Syt VII-deficient mice. The results revealed a marked defect in neurite outgrowth and arborization, suggesting that $\mathrm{Ca}^{2+}$-dependent, Syt VII-regulated exocytosis of late endosomes/lysosomes plays a role in the addition of new membrane to developing neurite extensions.
\end{abstract}

Key words: calcium; dendrite; development; exocytosis; fluorescence microscopy; knock-out mice

\section{Introduction}

The extension, branching, and interconnection of axons and dendrites play a central role in development of the nervous system (Prochiantz, 1995). Regrowth of neuritic trees is also observed during the regeneration of injured neurons, a process that is modulated by the influx of $\mathrm{Ca}^{2+}$ at the injury site (Rehder et al., 1992; Chu and Tator, 2001). Consistent with a mechanism based on regulated exocytosis, neurite outgrowth involves the polarized addition of new membrane at the tips of elongating processes (Craig et al., 1995; Futerman and Banker, 1996; Bradke and Dotti, 1997; Zakharenko and Popov, 1998; Martinez-Arca et al., 2001).

Vesicle-associated soluble $N$-ethylmaleimide-sensitive factor attachment protein receptor (SNARE) molecules present on in-

\footnotetext{
Received Jan. 3, 2006; revised March 17, 2006; accepted March 22, 2006.

This work was supported by National Institutes of Health Grant GM64625 (N.W.A.) and by a postdoctoral fellowship from Companha de Aperfeiçoamento de Pessoal de Nível Superior, Brazil to R.M.E.A. We thank Dr. Thierry Galli (Institut National de la Santé et de la Recherche Médicale, France) for the gift of anti-VAMP7 monoclonal antibodies, Christina Tam (Yale University) for preparation of anti-Syt VII affinity-purified antibodies, and Vincent Idone (Yale University) for maintenance of the Syt VII- / - mouse colony.

Correspondence should be addressed to Norma W. Andrews, Section of Microbial Pathogenesis, Boyer Center for Molecular Medicine, Yale University School of Medicine, 295 Congress Avenue, New Haven, CT 06510. E-mail: norma.andrews@yale.edu.

R. M. E. Arantes' present address: Department of General Pathology, Biological Sciences Institute, Federal University of Minas Gerais, Avenida Antonio Carlos, 6627-Pampulha, Caixa Postal 2486, Belo Horizonte, Minas Gerais 31270-010, Brazil.

DOI:10.1523/JNEUROSCI.0009-06.2006

Copyright $\odot 2006$ Society for Neuroscience $\quad$ 0270-6474/06/264630-08\$15.00/0
}

tracellular vesicles ( $\mathrm{v}$-SNARES) and target membranes ( $\mathrm{t}$ SNARES) are essential components of the molecular machinery responsible for membrane fusion (Rothman, 1994). Recent studies implicated the tetanus neurotoxin insensitive v-SNARE (TIVAMP)/VAMP7 in the exocytic events responsible for neurite extension. (Martinez-Arca et al., 2000). The $\mathrm{NH}_{2}$-terminal longin domain of VAMP7 inhibited SNARE complex formation and exerted dominant-negative effects on both axonal and dendritic outgrowth when introduced into neuroendocrine PC12 cells or primary neurons in culture (Martinez-Arca et al., 2000, 2001). Inhibition in neurite outgrowth was also observed after RNA interference-mediated silencing of VAMP7 (Alberts et al., 2003). These observations implicated VAMP7-containing intracellular compartments as a source of membrane for neurite outgrowth. However, the intracellular signaling pathways regulating VAMP7-dependent vesicular exocytosis and the nature of the VAMP7-containing compartments in neurons remain poorly understood.

VAMP7 was originally identified as a synaptobrevin-related SNARE (D'Esposito et al., 1996) resistant to cleavage by Tetanus and Botulinum neurotoxins (Galli et al., 1998). In fibroblasts, VAMP7 is localized on vesicular compartments containing the late endosomal/lysosomal glycoprotein 1 (Lamp1) (Advani et al., 1999; Rao et al., 2004) and mediates membrane traffic between late endosomes and lysosomes (Advani et al., 1999). Recently, VAMP7 was also shown to be required for $\mathrm{Ca}^{2+}$-triggered lyso- 
somal exocytosis in fibroblasts (Rao et al., 2004). After elevation in intracellular free $\mathrm{Ca}^{2+}\left(\left[\mathrm{Ca}^{2+}\right]_{\mathrm{i}}\right)$, VAMP7 forms SDS-resistant complexes with syntaxin 4 and soluble $N$-ethyl maleimidesensitive factor attachment protein 23 (SNAP23), two plasma membrane t-SNARES that interact in a $\mathrm{Ca}^{2+}$-dependent manner with the lysosomal synaptotagmin VII (Syt VII) (Rao et al., 2004). Syt VII is an evolutionarily conserved member of the synaptotagmin family of $\mathrm{Ca}^{2+}$ sensors (Andrews and Chakrabarti, 2005). Two $\mathrm{Ca}^{2+}$-binding C2 domains present on the cytosolic region of synaptotagmins mediate $\mathrm{Ca}^{2+}$-dependent interactions with membrane phospholipids and SNARE complex components, facilitating membrane fusion (Tucker and Chapman, 2002). Previous studies from our laboratory showed that Syt VII regulates $\mathrm{Ca}^{2+}$-triggered lysosomal exocytosis (Martinez et al., 2000) and the resealing of plasma membrane wounds (Reddy et al., 2001). The functional association of Syt VII with VAMP7 observed during lysosomal exocytosis in fibroblasts (Rao et al., 2004) suggested that similar $\mathrm{Ca}^{2+}$-regulated exocytic events might be involved in neurite outgrowth. We investigated this possibility using primary neurons isolated from mouse sympathetic cervical ganglia (SCGs). Our results show that VAMP7 and Syt VII colocalize in peripheral late endosomal/lysosomal compartments that exocytose after $\left[\mathrm{Ca}^{2+}\right]_{i}$ elevation, and that genetic ablation of Syt VII results in defective neurite outgrowth.

\section{Materials and Methods}

Materials. Monoclonal anti-mouse Lamp1 antibodies (1D4B) were obtained from the Developmental Studies Hybridoma Bank (Iowa City, IA), and monoclonal anti-VAMP2 (C169.1) was obtained from Synaptic Systems (Goettingen, Germany). Polyclonal rabbit antibodies against the luminal NH2-terminal sequence of synaptotagmin VII were generated and affinity-purified as described previously (Martinez et al., 2000). Monoclonal antibodies (mAbs) (clone 158.2) against TI-VAMP/VAMP7 were a generous gift from Dr. Thierry Galli (Institut National de la Santé et de la Recherche Médicale U536, Institut du Fer-'a-Moulin, Paris, France). Fluorescent secondary Alexa Fluor 488 or 546 antibodies were purchased from Invitrogen (Carlsbad, CA), and ionomycin was purchased from Calbiochem (San Diego, CA). Mouse nerve growth factor (NGF 2, 5S) was purchased from Alomone Labs (Jerusalem, Israel), and arabinoside C was purchased from Sigma (St. Louis, MO).

Immunofluorescence. Localization of antigens of interest were performed on monolayers fixed with $2 \%$ paraformaldehyde (PFA). After permeabilization with $0.1 \%$ saponin for $15 \mathrm{~min}$, the cells were incubated with 1:100 dilutions of antibodies against LAMP1, TI-VAMP/VAMP7, and VAMP2, followed by the appropriate fluorescent secondary antibodies. For staining with antibodies against synaptotagmin VII (1:100 dilution), cells were permeabilized with $0.1 \%$ Triton X-100. Double immunohistochemistry for observation of colocalization of the markers was performed with an initial $0.1 \%$ saponin permeabilization in the case Lamp 1 staining, followed by permeabilization in $0.05 \%$ Triton X-100 for TI-VAMP/VAMP7 or Syt VII staining. Secondary antibodies alone were used as specificity controls and uniformly resulted in very low background levels of fluorescence.

Detection of surface Lamp1 and Syt VII after exocytosis. Neuronal cultures were washed and incubated at $37^{\circ} \mathrm{C}$ with $0.5 \mathrm{ml}$ of either PBS alone or PBS containing $5 \mu \mathrm{M}$ ionomycin for $3-5 \mathrm{~min}$. The cells were then incubated at $4^{\circ} \mathrm{C}$ for $30 \mathrm{~min}$ with anti-LAMP1 (1D4B hybridoma culture supernatant) or anti-Syt VII luminal domain antibodies. Cells were then fixed with $2 \%$ PFA for $15 \mathrm{~min}$ at $4^{\circ} \mathrm{C}$, washed in PBS, and incubated with the appropriate secondary antibodies for $30 \mathrm{~min}$ at room temperature. Nuclei were stained with $4^{\prime}, 6^{\prime}$-diamidino-2-phenylindole (DAPI) (Sigma).

SCG explant assay. Syt VII +/+ and Syt VII-/- mice were generated as described previously (Chakrabarti et al., 2003) and back-crossed for eight generations into the C57BL/6 background. Superior SCGs were bilaterally dissected from newborn (1-2 d of age) Syt VII+/+ or Syt
VII-/ - mice, washed in ice-cold PBS, and explanted to Matrigel-coated $35 \mathrm{~mm}$ glass-bottom culture dishes (Matek Corporation, Ashland, MA). A drop of cold Matrigel (BD Biosciences, PharMingen, San Diego CA) diluted 1:4 in DMEM was placed at the bottom of the dish and incubated at room temperature until the gel solidified. Each dissected SCG cleaned of all surrounding connective tissue was deposited on the top of the drop, additional $20 \mu \mathrm{l}$ of diluted Matrigel was added, and the dish was incubated for $15 \mathrm{~min}$ at room temperature. DMEM (1.5 ml) 10\% FCS supplemented with $50 \mathrm{ng} / \mathrm{ml} \mathrm{NGF}$ (Alomone Labs) was then added, and the explants were incubated for $3,12,24,72$, and $120 \mathrm{~h}$ at $37^{\circ} \mathrm{C} 5 \% \mathrm{CO}_{2}$. At each time point, phase contrast images of SCG explants were acquired using a $10 \times$ objective $(100 \times$ final magnification) of a Zeiss (Thornwood, NY) Axiovert 135 microscope equipped with a Hamamatsu (Shizuoka, Japan) Orca II cooled CCD camera controlled by MetaMorph software (Molecular Devices, Sunnyvale, CA).

Explant outgrowth measurements. For the early time points (3-24 h), explants were small enough to be visualized and measured in a single microscopic field. After this initial growth period, four separate images covering the complete ganglion were taken from each explant, and the outgrowth length was determined on scanned images of each quadrant captured with a $10 \times$ objective. Measurements were made in exported eight-bit TIFF files using the image analysis software MetaMorph. Maximum neurite length was determined as follows: the MetaMorph "draw" function was used to draw a straight line from the border of the explants to the tip of the longest neurite or neurite bundle at 30 different locations covering each of the four quadrants of explants, as described previously (Weaver et al., 2003). Maximum neurite length was defined as the average of these 30 measurements, and the values were calibrated in micrometers using the MetaMorph "calibrate" function. In each experiment, the minimum number of ganglia measured per time point was three, and experiments were repeated three to five times. The results were expressed as the average and SD of the results of each separate experiment and analyzed for statistical significance with an unpaired Student's $t$ test ( $p$ values $<0.05$ were considered significant).

Isolated neuron cultures. Primary neuronal cultures were prepared from SCGs of newborn Syt VII+/+ or Syt VII-/- mice, as described previously (Arantes et al., 2000). SCGs were bilaterally dissected from newborn (1-2 d of age) C57BL/6 mice (average of 16 ganglia per litter), washed in PBS, and combined in a single pool. The cells were incubated for $1 \mathrm{~h}$ at $37^{\circ} \mathrm{C}$ in $0.25 \%$ trypsin solution (Invitrogen, Carlsbad, CA) followed by mechanical dissociation with a long-tip, fire-polished Pasteur pipette until individual cells could be visualized and counted in a Neubauer chamber. Cells were plated at $10^{4} /$ dish on glass-bottom 35 mm dishes previously coated with Matrigel diluted 1:4 in DMEM (Invitrogen) and grown in DMEM 10\% FBS containing $50 \mathrm{ng} / \mathrm{ml} \mathrm{NGF}$ (required for neuronal survival) and $0.1 \mathrm{~mm}$ arabinoside $\mathrm{C}$ (for inhibiting the growth of glia and other accessory cells) for the entire experimental period. Cultures were kept at $37^{\circ} \mathrm{C}$ in $5 \% \mathrm{CO}_{2}$ for $1,2.5,4$, and $24 \mathrm{~h}$, followed by fixation in 2\% PFA for $30 \mathrm{~min}$ at room temperature.

Quantification of neurite outgrowth in isolated neurons. Images of randomly chosen fields of fixed SCG isolated neuron cultures were obtained under $1000 \times$ magnification using a Zeiss Axiovert 135 microscope equipped with a Hamamatsu Orca II cooled CCD camera and MetaMorph software. Images from at least 60 individual fields were captured, resulting in the analysis of 50-150 neurons per time point, per experiment. Neurons were distinguished from other cell types by immunofluorescence with antibodies against VAMP2. Quantification of neurite length was done on exported eight-bit TIFF files using the MetaMorph image analysis software. Neurites were outlined using the draw function, and their perimeter was automatically measured and calibrated in micrometers using the MetaMorph calibrate function. The number of primary neurites (defined as the segments of neurites originating directly from the cell body of neurons) and the number of branching points were microscopically counted. For each microscopic field, the number of neuronal cell bodies was also counted, and the data were expressed as the average neurite length/neuron, number of primary neurites/neuron, and branching points/neuron. Because at $24 \mathrm{~h}$ the neurite network was too complex to allow accurate branch point measurements, at this time point, neurite density was determined by superposing two vertical lines 
on the captured images, as described previously (Arantes et al., 2000; Ronn et al., 2000). Neurite density was defined as the average number of neurites that were intersected by the lines. The results were expressed as the average and SD of three independent experiments and analyzed for statistical significance with an unpaired Student's $t$ test ( $p$ values $<0.05$ were considered significant).

\section{Results}

Late endosomes/lysosomes containing Lamp1 and VAMP7 are present in neurite outgrowths, including the tips of elongating processes

Previous studies concluded that the late endosomes and lysosomes of primary neurons were predominantly located in the soma and proximal dendrites (Parton et al., 1992; Craig and Banker, 1994). Subsequent work using more sensitive immunofluorescence techniques revealed the presence of lysosomes throughout axon shafts, including branching points (Overly and Hollenbeck, 1996). However, it remained unclear whether late endosomes/lysosomes were present at sites of new membrane addition, such as the tips of elongating neurites during neuronal development. To investigate this issue, we performed immunofluorescence with antibodies against the lysosomal marker Lamp1 on SCG neurons at increasing time periods after plating dissociated ganglia in the presence of NGF. After $1-2 \mathrm{~h}$, the culture contained multiple clusters of neurons (identified by labeling with anti-synaptotagmin I and anti-VAMP2 antibodies) (Fig. $2 B$ ), some of which contained short neurite extensions (Fig. 1A). Although Lamp1-positive compartments were abundant in cell bodies, clusters of vesicles reactive with the antibody were also detected at distal regions of the elongating neurites (Fig. $1 B$ ). The presence of lysosomes within growing neurites was more evident after 4 h of culture, when Lamp1-positive vesicles were frequently observed at branching points and at the tips of elongating processes (Fig. 1C-E).

Previous studies demonstrated a role for the late endosomal v-SNARE VAMP7 in neurite outgrowth from PC12 cells and primary hippocampal neurons (Martinez-Arca et al., 2000, 2001; Alberts et al., 2003). Although VAMP7 was found to colocalize with the late endosomal/lysosomal marker CD63 (Coco et al., 1999), the late endosomal/lysosomal nature of the VAMP7 compartments was questioned in those studies because of an apparent lack of VAMP7 colocalization with Lamp1 (Coco et al., 1999). To clarify this issue, we performed double immunofluorescence assays with antibodies against Lamp1 and VAMP7 on developing SCG neurons. We detected extensive colocalization between these two markers, both at early (Fig. 2A, $1 \mathrm{~h}$ ) and later (Fig. $2 \mathrm{~A}$, $4 \mathrm{~h}$ ) time points. Vesicles immunoreactive for both Lampl and VAMP7 were present throughout the neurons, and clusters of vesicles labeled by both antibodies were frequently observed within growth cones (Fig. $2 A, 4 \mathrm{~h}$ ). The synaptic vesicle SNARE VAMP2 was also detected within growth cone regions, but its localization was clearly distinct from the lysosomal marker Lamp1 (Fig. 2 B). Thus, these results demonstrate that in primary sympathetic neurons, VAMP7-containing compartments completely overlap with Lamp1-positive late endosomes/lysosomes, as shown previously in other cell types (Advani et al., 1999; Rao et al., 2004).

The lysosomal synaptotagmin Syt VII is present in Lamp1positive compartments of SCG neurons and translocated to the cell surface after $\mathrm{Ca}^{2+}$-triggered exocytosis

Syt VII was previously shown to colocalize with lysosomal markers in fibroblasts (Martinez et al., 2000; Caler et al., 2001; Jaiswal
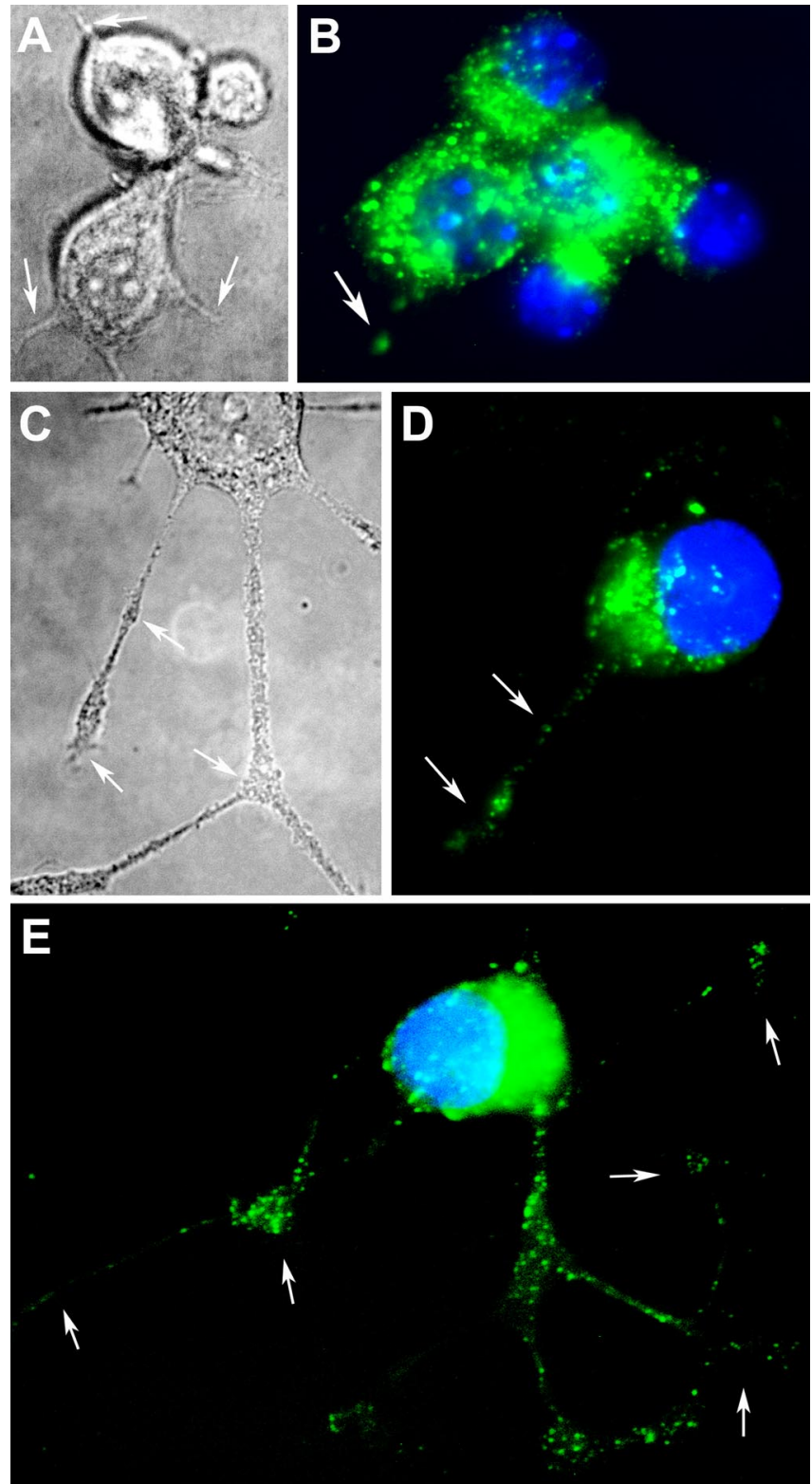

Figure 1. Lamp1-containing vesicles are present in the cell body and throughout neuritic outgrowths of SCG neurons. A, C, DIC images of SCG neurons at $2.5 \mathrm{~h}(\boldsymbol{A})$ or $4 \mathrm{~h}(\boldsymbol{C})$ after plating, showing the typical pyramidal, globular shape of the cells with thin extensions originating from the soma (arrows). $\boldsymbol{B}, \boldsymbol{D}, \boldsymbol{E}$, Immunofluorescence of saponin-permeabilized SCG neurons with anti-Lamp $1 \mathrm{mAbs}$ (green) and nuclear DAPI staining (blue) after $1 \mathrm{~h}(\boldsymbol{B})$ and $4 \mathrm{~h}(\boldsymbol{D}, \boldsymbol{E})$ in culture. Arrows point to distal regions and branching points of growing neurites, where Lamp1-positive vesicles were detected $(\boldsymbol{D}, \boldsymbol{E})$.

et al., 2002; Chakrabarti et al., 2003), epithelial cells (Caler et al., 2001), keratinocytes (Hakansson et al., 2005), and the neuroendocrine cell line PC12 (Fukuda et al., 2004; Wang et al., 2005). To determine whether the same occurred in primary neurons, we performed immunolocalization of Syt VII in isolated SCG neurons. These assays revealed the presence of intracellular vesicular compartments containing both Lamp1 and Syt VII, within the cell bodies and also at peripheral regions associated with developing neurite extensions (Fig. 3). The relative abundance of Lamp1 and Syt VII on the double-positive vesicles detected varied, suggesting the existence of Syt VII or Lamp1-enriched domains on the same compartments. 

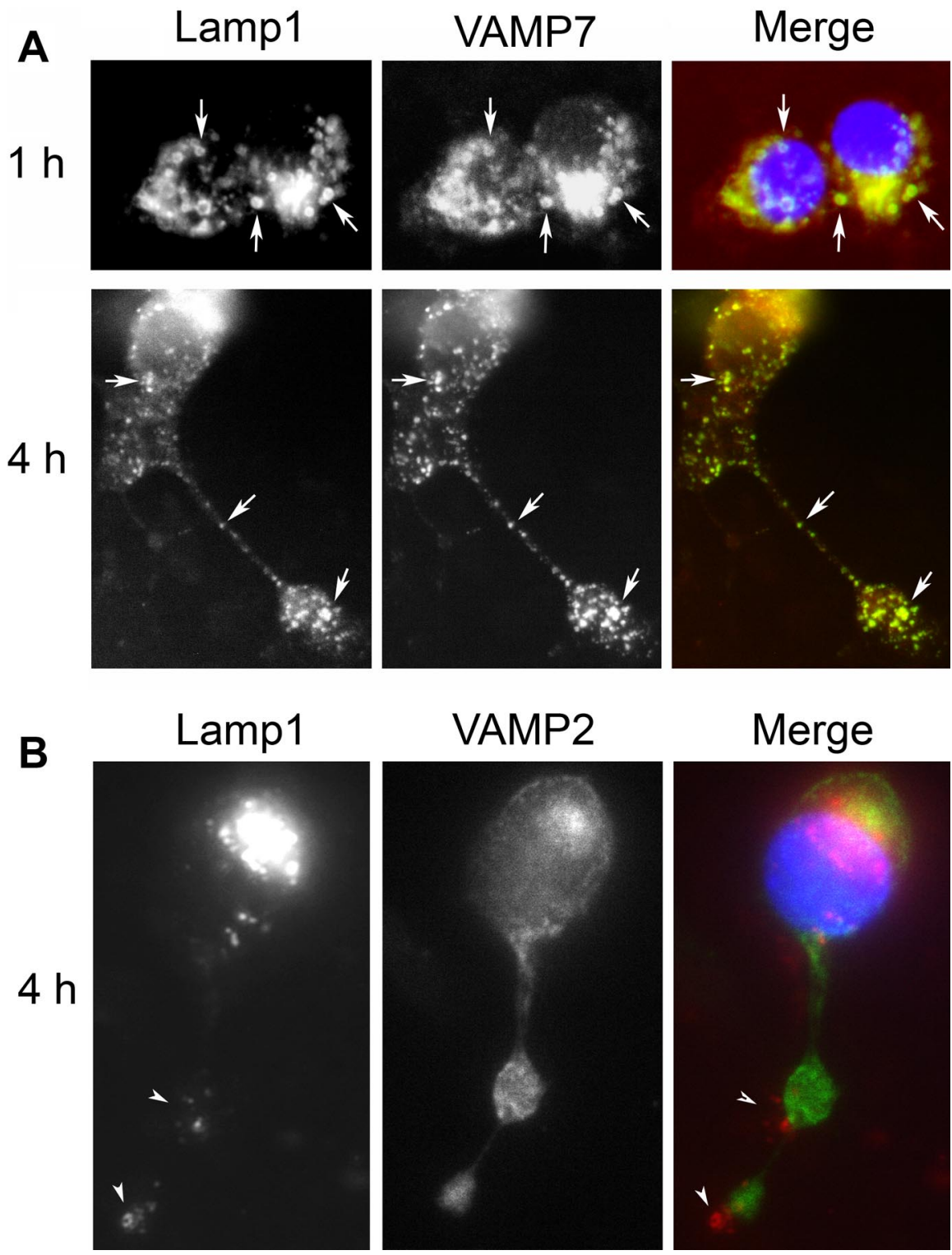

Figure 2. Lamp1 colocalizes with VAMP7 but not VAMP2 in developing SCG neurons. A, Immunofluorescence of permeabilized SCG neurons with $\mathrm{mAbs}$ against Lamp1 (green in merged image) and VAMP7 (red in merged image) after $1 \mathrm{~h}$ (top) or $4 \mathrm{~h}$ (bottom) in culture. Arrows point to vesicles containing both Lamp1 and VAMP7. B, Immunofluorescence of permeabilized SCG neurons with mAbs against Lamp1 (red in merged image) and VAMP2 (green in merged image) after $4 \mathrm{~h}$ in culture. Arrowheads point to Lamp1-positive vesicle clusters at distal regions of the growing neurite, which do not colocalize with VAMP2-positive synaptic vesicles.

Previous studies from our laboratory showed that the luminal domains of Lamp1 and Syt VII are exposed on the surface of fibroblasts, when lysosomal exocytosis is induced by elevation in $\left[\mathrm{Ca}^{2+}\right]_{\mathrm{i}}$ (Reddy et al., 2001). To determine whether the lysosomal exocytic pathway was functional in SCG neurons, we exposed cultures of developing neurons to the $\mathrm{Ca}^{2+}$ ionophore ionomycin and performed immunofluorescent detection of surfaceexposed Lamp1 and Syt VII. As reported previously for fibroblasts (Rodriguez et al., 1997; Reddy et al., 2001), elevation in $\left[\mathrm{Ca}^{2+}\right]_{\mathrm{i}}$ resulted in a punctate labeling pattern on the surface of SCG neurons, when intact cells were surface-stained with antibodies against the luminal domain of Lamp1 (Fig. 4). Several of the surface-exposed, Lamp1-positive puncta colocalized precisely with areas where the luminal domain of Syt VII was also detected with specific antibodies. Spots containing both Lamp1 and Syt VII were detected predominantly at the periphery of developing neurons and, in some instances, appeared to be associated with sites of neurite outgrowth (Fig. 4). Consistent with the fact that not all Lamp1positive intracellular compartments in SCG neurons contain detectable Syt VII (Fig. 3), a fraction of the surface-exposed luminal domain of Lamp1 was not associated with Syt VII and was detected in a more perinuclear location (Fig. 4). These observations suggested that lysosomal compartments of sympathetic neurons are capable of fusing with the plasma membrane after $\left[\mathrm{Ca}^{2+}\right]_{\mathrm{i}}$ elevation, and that a more peripheral fraction of these Lamp1positive exocytic compartments contains the $\mathrm{Ca}^{2+}$ sensor Syt VII.

\section{Neurite outgrowth is impaired in SCG} neurons from Syt VII-deficient mice Previous studies in fibroblasts demonstrated that genetic ablation of Syt VII inhibits lysosomal exocytosis, when the process is triggered by $\mathrm{Ca}^{2+}$ influx at sites of membrane injury (Chakrabarti et al., 2003; Roy et al., 2004) or by mobilization of intracellular $\mathrm{Ca}^{2+}$ stores through receptor-mediated agonists (Caler et al., 2001; Jaiswal et al., 2004). In contrast, the higher and more widespread $\left[\mathrm{Ca}^{2+}\right]_{\mathrm{i}}$ elevation resulting from stimulation with $\mathrm{Ca}^{2+}$ ionophores can also trigger lysosomal exocytosis in Syt VII-deficient cells, albeit with a markedly altered secretory pattern when compared with wild-type cells (Jaiswal et al., 2004). However, concerns about toxic and ATP-depleting effects of $\mathrm{Ca}^{2+}$ ionophores has limited the usefulness of this procedure for the study of physiologically relevant exocytosis (Andrews and Chakrabarti, 2005). For this reason, we examined the consequences of Syt VII ablation using physiological assays of NGF-dependent neurite outgrowth, a process already shown to require the exocytosis of intracellular compartments containing VAMP7 (Martinez-Arca et al., 2000, 2001).

SCG ganglia isolated from Syt VII+/+ or Syt VII-/- mice were cultured within Matrigel matrices in the presence of NGF for increasing periods of time, and the average length of neurites outgrowing from the explants was determined on microscopically acquired images. In independent experiments, a reduction in neurite outgrowth was evident in ganglion explants from Syt VII $-/-$ mice after $12-120 \mathrm{~h}$ of culture (Fig. $5 A, B$ ).

To characterize more precisely the apparent defect in neurite outgrowth resulting from Syt VII deficiency, we examined isolated SCG neurons during their initial $24 \mathrm{~h}$ of development in culture. Isolated ganglia were dissociated, and the isolated neurons were plated in Matrigel-coated dishes in the presence of 

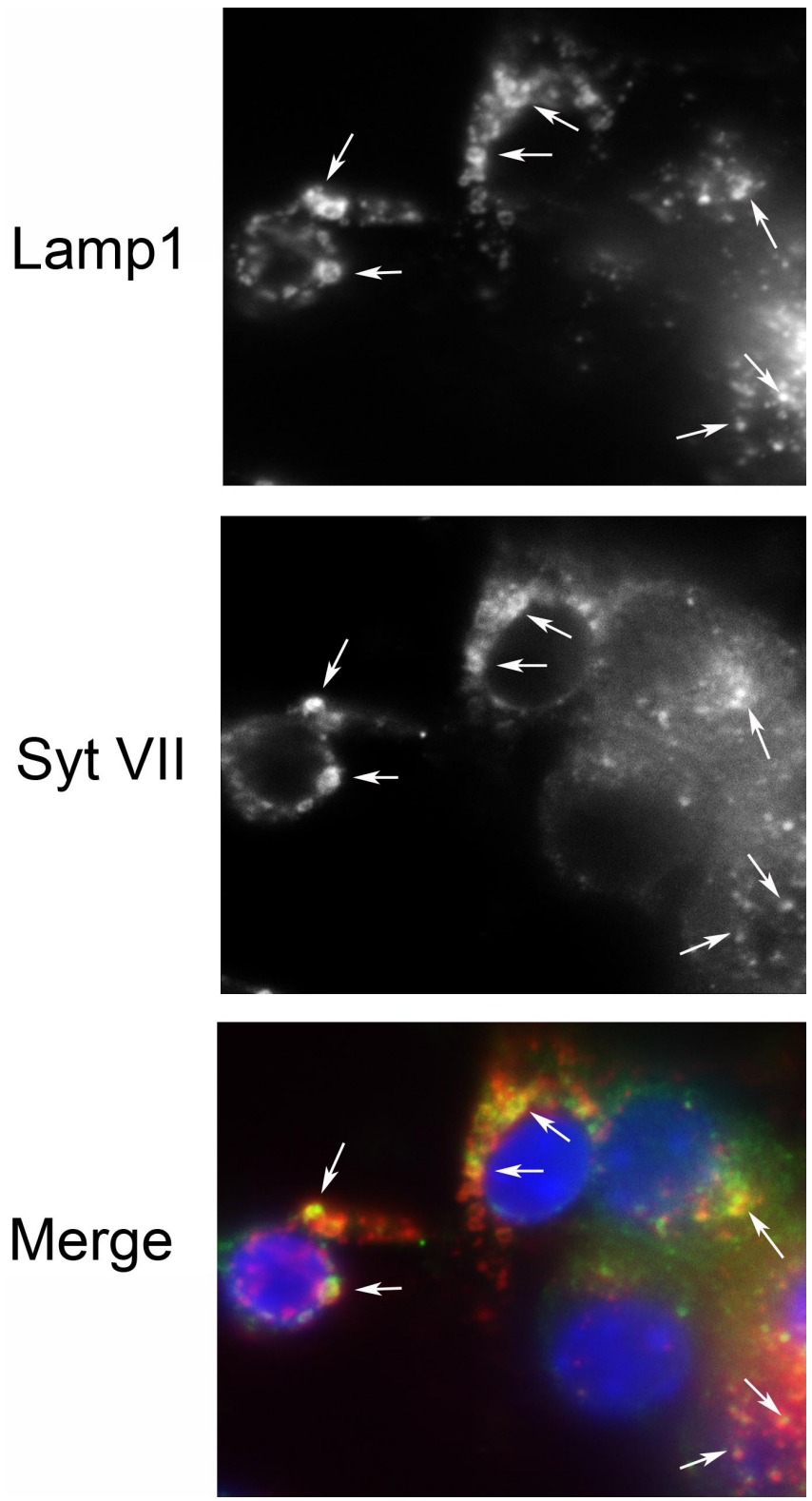

Figure 3. Syt VII is present in Lamp1-positive compartments of developing SCG neurons. Immunofluorescence was performed on saponin-permeabilized SCG neurons after $2.5 \mathrm{~h}$ in culture with anti-Lamp1 mAbs and anti-Syt VII affinity-purified polyclonal antibodies. Arrows point to compartments containing both Lamp1 (red in merged image) and Syt VII (green in merged image). Nuclei in merged image were stained with DAPI (blue).

NGF and fixed and imaged after 1, 2.5, 4, and $24 \mathrm{~h}$. Although there was a reduction in the number of neurons remaining attached to the substrate between 4 and $24 \mathrm{~h}$, the loss was similar in Syt VII+ / + and Syt VII-/ - cultures (supplemental Fig. 1, available at www.jneurosci.org as supplemental material). The images were analyzed for average neurite length (total area occupied by extensions emanating from the cell bodies), number of primary neurites, and number of branching points (Figs. 6, 7). Statistically significant differences were observed between Syt VII $+/+$ and Syt VII-/- neurons in three independent experiments. The average neurite length was markedly reduced in Syt VII-/- neurons after $24 \mathrm{~h}$ in culture (Fig. 7A), and significant decreases in the number of primary neurites and in the number of branching points were also detected after $1,2.5,4$, and $24 \mathrm{~h}$ (Fig. $7 B-D$ ).
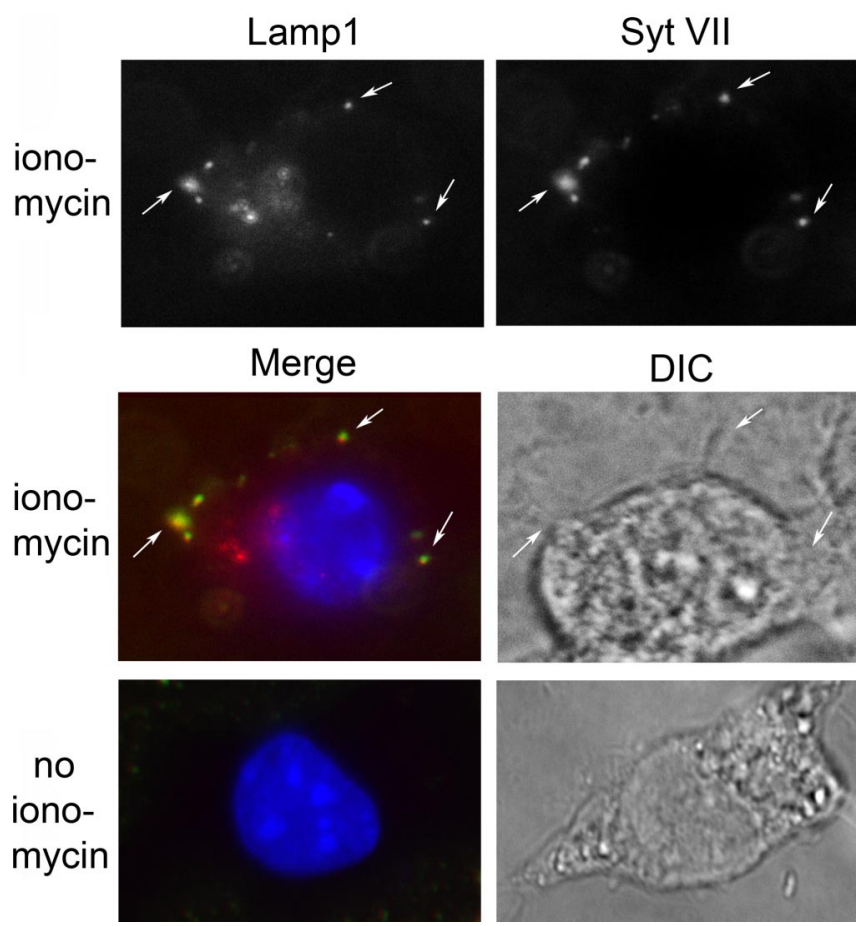

Figure 4. The luminal domain of Lamp1 and Syt VII colocalize on the surface of SCG neurons after $\mathrm{Ca}^{2+}$-triggered exocytosis. Intact SCG neurons cultured for $2.5 \mathrm{~h}$ were exposed or not to ionomycin and surface labeled at $4^{\circ} \mathrm{C}$ with antibodies against luminal epitopes of Lamp 1 (red in merged image) and Syt VII (green in merged image). Arrows point to regions of coexposure of the luminal regions of Lamp1 and Syt VII on the neuronal surface. The DIC images on the right side of the bottom panel correspond to the same neurons shown on the left.

Together, these results indicate that the late endosomal/lysosomal $\mathrm{Ca}^{2+}$ sensor Syt VII participates in the exocytic process mediating neurite outgrowth. Syt VII absence from SCG neurons results in a defect in new membrane deposition and in a markedly reduced complexity of the arborification process of developing growth cones.

\section{Discussion}

Previous studies demonstrated that intracellular compartments containing the V-SNARE VAMP7 participate in the exocytic delivery of intracellular membrane to neurite extension sites (Martinez-Arca et al., 2000, 2001; Alberts et al., 2003). It remained unclear, however, which were the properties of these VAMP7-positive compartments that make them responsive to signals regulating localized exocytosis. In this study, we show that VAMP7 and the lysosomal marker Lamp1 fully overlap in primary sympathetic SCG neurons. These VAMP7/Lamp1-positive late endosomes/lysosomes are also present within growth cones at distal regions of developing neuronal processes. A subset of these vesicles contains Syt VII, the ubiquitously expressed synaptotagmin isoform previously shown to regulate $\mathrm{Ca}^{2+}$-triggered exocytosis of lysosomes. We found that Syt VII/VAMP7-positive vesicles in SCG neurons behave as $\mathrm{Ca}^{2+}$-regulated secretory vesicles, fusing with the plasma membrane after $\left[\mathrm{Ca}^{2+}\right]_{\mathrm{i}}$ elevation. These findings suggested that Syt VII-dependent $\mathrm{Ca}^{2+}$-triggered exocytosis might be involved in the mobilization of late endosomes/lysosomes to the plasma membrane during neurite outgrowth. We examined this question by following the development in culture of SCG neurons isolated from wild-type or Syt VII-deficient mice. Our results show that the number, length, 
A

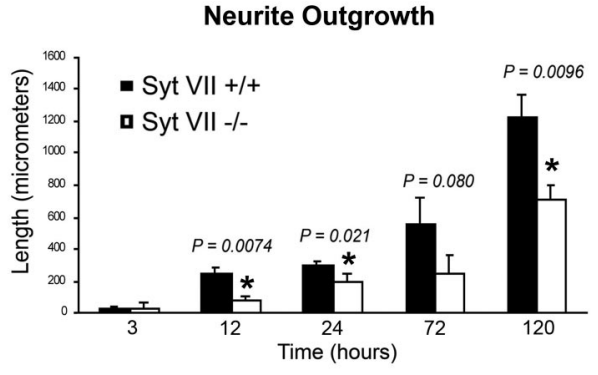

B Syt VII +/+

$24 \mathrm{~h}$
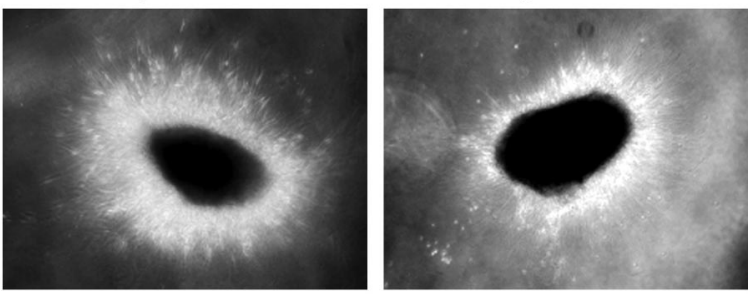

$120 \mathrm{~h}$
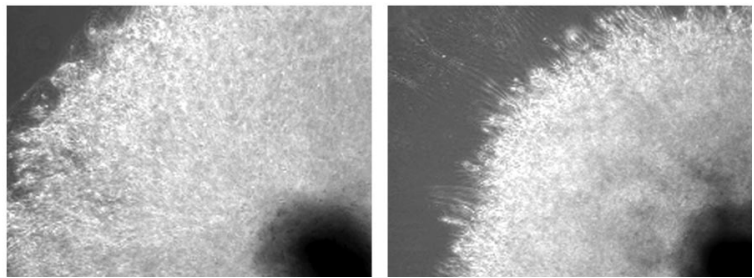

Figure 5. Neurite outgrowth is impaired in SCG explants from Syt VII-deficient mice. $\boldsymbol{A}$ Average neurite length after increasing periods of time in culture. The data are expressed as the mean \pm SD of neurite length average values from independent experiments $(n=3-5)$. $\boldsymbol{B}$, Phase contrast images of SCG explants after 24 and $120 \mathrm{~h}$ in culture, illustrating the reduced neurite outgrowth observed in Syt VII- / - neurons (right panels).

and complexity of neuritic tree outgrowths are significantly reduced in Syt VII-deficient neurons, directly implicating the $\mathrm{Ca}^{2+}$ sensor Syt VII in this process.

Several studies have suggested a role for $\left[\mathrm{Ca}^{2+}\right]_{\mathrm{i}}$ elevation as a trigger for neurite outgrowth. In addition to being required for membrane resealing in injured neurites, $\mathrm{Ca}^{2+}$ influx induces the formation of growth cones (Davenport and Kater, 1992; Rehder et al., 1992; Ziv and Spira, 1997; Chu and Tator, 2001; Shoemaker et al., 2005). A few minutes after membrane injury or the local application of $\mathrm{Ca}^{2+}$ ionophores, Aplysia giant neuron axonal segments dedifferentiate into growth cones and generate a vigorous neuritic tree outgrowth (Ziv and Spira, 1997). Similar observations were made in rat SCG neurons; transected neurites were able to regrow in the presence of extracellular $\mathrm{Ca}^{2+}$ but not when neurons were preloaded with the $\mathrm{Ca}^{2+}$ chelator BAPTA-AM (Chu and Tator, 2001). In our experimental system, mouse SCG neurons were cultured in the presence of NGF, which is essential for their long-term survival. NGF exerts its neurotrophic action through Trk receptors (Kalb, 2005), which when activated can lead to small and rapid increases in $\left[\mathrm{Ca}^{2+}\right]_{\mathrm{i}}($ Pandiella-Alonso et al., 1986; Jiang and Guroff, 1997). Basal $\left[\mathrm{Ca}^{2+}\right]_{\mathrm{i}}$ levels seem to be important for the NGF-dependent survival of sympathetic neurons in culture (Koike and Tanaka, 1991), but it remains unclear whether NGF-dependent $\mathrm{Ca}^{2+}$ signaling is required for neurite outgrowth (Tolkovsky et al., 1990). Although the neuritic tree outgrowth observed in our studies may have been, at least in part, triggered by $\mathrm{Ca}^{2+}$ influx through wounds on neurite membranes generated during SCG isolation, a role for NGF-dependent
$1 \mathrm{~h}$
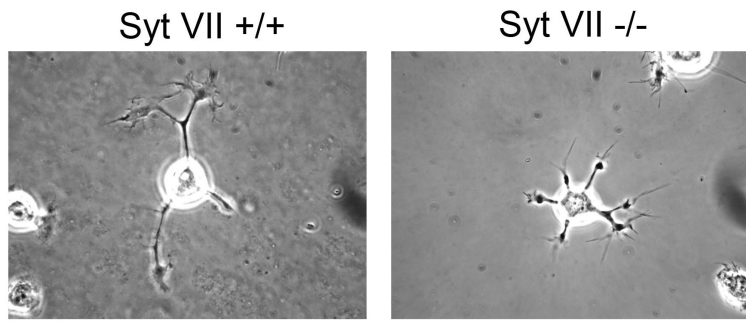

$2.5 \mathrm{~h}$
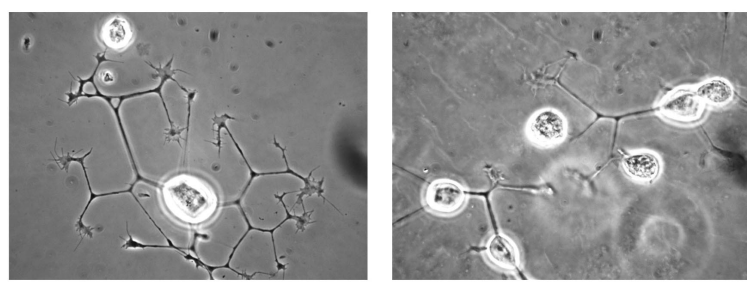

$4 \mathrm{~h}$
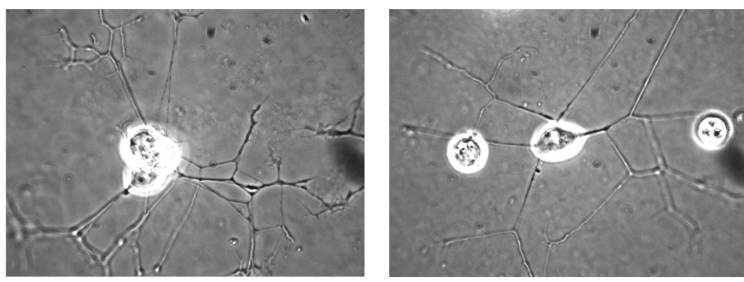

$24 \mathrm{~h}$
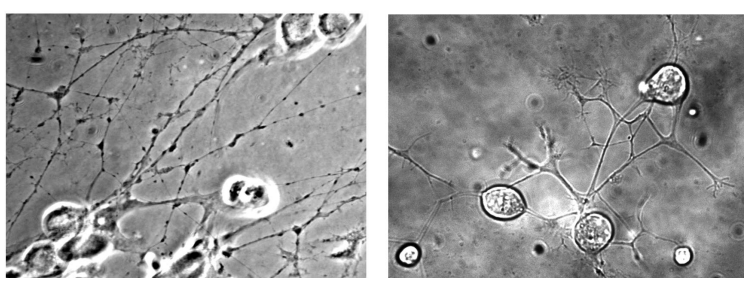

Figure 6. Neurite growth is impaired in neurons isolated from SCG explants from Syt VIIdeficient mice. Phase contrast images of isolated $S C G$ neurons after increasing periods of time in culture show the reduced complexity of the outgrowing neuritic trees in Syt $\mathrm{VII}-/-$ neurons.

$\left[\mathrm{Ca}^{2+}\right]_{\mathrm{i}}$ increases cannot be ruled out. Because NGF is required for long-term survival of SCG neurons in culture, it has not been possible to directly examine the role of NGF as a mediator of $\mathrm{Ca}^{2+}$ signaling and of Syt VII-dependent neurite extension.

Axonal growth and dendrite extension are critical events in the development of the nervous system. Despite the marked defect observed in neurite outgrowth from explanted SCG neurons in culture, Syt VII deficiency does not result in abnormal brain development or in any obvious neurological phenotype (Chakrabarti et al., 2003). A similar lack of a brain development phenotype was observed in SNAP25 mouse null mutants (Washbourne et al., 2002), in contrast to the strong inhibition in neurite outgrowth seen in isolated hippocampal neurons after SNAP25 cleavage with Botulinum neurotoxin A (Grosse et al., 1999). The lack of a direct correlation between in vitro and in vivo phenotypes is often related to the existence of functionally redundant pathways. This could have occurred at the level of the molecules mediating vesicular traffic (such as additional synaptotagmin isoforms in Syt VII-/- mice, or the functional replacement of SNAP25 by SNAP23) or at the level of the signaling pathways triggering neurite extension. One example of a redundant signaling pathway is the inhibition in neurite outgrowth observed in Ryk-deficient dorsal root ganglion (DRG) neurons stimulated with Wnt, but not with NGF, providing an explanation for the apparently normal in vivo DRG axon outgrowth of Ryk-deficient mice (Lu et al., 2004). It will be of interest to determine whether 
the impaired neuritic outgrowth we observed in cultured Syt VII-deficient SCG neurons has an impact in the postnatal maintenance of dendritic arbor structure in vivo. Recent evidence suggests that dendrite branch maintenance may be essential for sustaining normal brain function (Zoghbi, 2003; Moresco et al., 2005).

\section{References}

Advani RJ, Yang B, Prekeris R, Lee KC, Klumperman J, Scheller RH (1999) VAMP-7 mediates vesicular transport from endosomes to lysosomes. J Cell Biol 146:765-776.

Alberts P, Rudge R, Hinners I, Muzerelle A, Martinez-Arca S, Irinopoulou T, Marthiens V, Tooze S, Rathjen F, Gaspar P, Galli T (2003) Cross talk between tetanus neurotoxin-insensitive vesicle-associated membrane protein-mediated transport and L1mediated adhesion. Mol Biol Cell 14:4207-4220.

Andrews NW, Chakrabarti S (2005) There's more to life than neurotransmission: the regulation of exocytosis by synaptotagmin VII. Trends Cell Biol 15:626-631.

Arantes RM, Lourenssen S, Machado CR, Blennerhassett MG (2000) Early damage of sympathetic neurons after co-culture with macrophages: a model of neuronal injury in vitro. NeuroReport 11:177-181.

Bradke F, Dotti CG (1997) Neuronal polarity: vectorial cytoplasmic flow precedes axon formation. Neuron 19:1175-1186.

Caler EV, Chakrabarti S, Fowler KT, Rao S, Andrews NW (2001) The exocytosis-regulatory protein synaptotagmin VII mediates cell invasion by Trypanosoma cruzi. J Exp Med 193:1097-1104.

Chakrabarti S, Kobayashi KS, Flavell RA, Marks CB, Miyake K, Liston DR, Fowler KT, Gorelick FS, Andrews NW (2003) Impaired membrane resealing and autoimmune myositis in synaptotagmin VII-deficient mice. J Cell Biol 162:543-549.

Chu GK, Tator CH (2001) Calcium influx is necessary for optimal regrowth of transected neurites of rat sympathetic ganglion neurons in vitro. Neuroscience 102:945-957.

Coco S, Raposo G, Martinez S, Fontaine JJ, Takamori S, Zahraoui A, Jahn R, Matteoli M, Louvard D, Galli T (1999) Subcellular localization of tetanus neurotoxin-insensitive vesicle-associated membrane protein (VAMP)/VAMP7 in neuronal cells: evidence for a novel membrane compartment. J Neurosci 19:9803-9812.

Craig AM, Banker G (1994) Neuronal polarity. Annu Rev Neurosci 17:267-310.

Craig AM, Wyborski RJ, Banker G (1995) Preferential addition of newly synthesized membrane protein at axonal growth cones. Nature 375:592-594.

D’Esposito M, Ciccodicola A, Gianfrancesco F, Esposito T, Flagiello L, Mazzarella R, Schlessinger D, D’Urso M (1996) A synaptobrevin-like gene in the Xq28 pseudoautosomal region undergoes X inactivation. Nat Genet 13:227-229.

Davenport RW, Kater SB (1992) Local increases in intracellular calcium elicit local filopodial responses in Helisoma neuronal growth cones. Neuron 9:405-416.

Fukuda M, Kanno E, Satoh M, Saegusa C, Yamamoto A (2004) Synaptotagmin VII is targeted to dense-core vesicles and regulates their $\mathrm{Ca}^{2+}$ dependent exocytosis in PC12 cells. J Biol Chem 279:52677-52684.

Futerman AH, Banker GA (1996) The economics of neurite outgrowth-the addition of new membrane to growing axons. Trends Neurosci 19:144-149.

Galli T, Zahraoui A, Vaidyanathan VV, Raposo G, Tian JM, Karin M, Niemann H, Louvard D (1998) A novel tetanus neurotoxin-insensitive vesicle-associated membrane protein in SNARE complexes of the apical plasma membrane of epithelial cells. Mol Biol Cell 9:1437-1448.

Grosse G, Grosse J, Tapp R, Kuchinke J, Gorsleben M, Fetter I, Hohne-Zell B, Gratzl M, Bergmann M (1999) SNAP-25 requirement for dendritic growth of hippocampal neurons. J Neurosci Res 56:539-546.

Hakansson A, Bentley CC, Shakhnovic EA, Wessels MR (2005) Cytolysindependent evasion of lysosomal killing. Proc Natl Acad Sci USA 102:5192-5197.
Jaiswal JK, Andrews NW, Simon SM (2002) Membrane proximal lysosomes are the major vesicles responsible for calcium-dependent exocytosis in nonsecretory cells. J Cell Biol 159:625-635.

Jaiswal JK, Chakrabarti S, Andrews NW, Simon SM (2004) Synaptotagmin VII restricts fusion pore expansion during lysosomal exocytosis. PLoS Biol 2:1224-1232.

Jiang H, Guroff G (1997) Actions of the neurotrophins on calcium uptake. J Neurosci Res 50:355-360.

Kalb R (2005) The protean actions of neurotrophins and their receptors on the life and death of neurons. Trends Neurosci 28:5-11.

Koike T, Tanaka S (1991) Evidence that nerve growth factor dependence of sympathetic neurons for survival in vitro may be determined by levels of cytoplasmic free Ca2+. Proc Natl Acad Sci USA 88:3892-3896.

Lu W, Yamamoto V, Ortega B, Baltimore D (2004) Mammalian Ryk is a Wnt coreceptor required for stimulation of neurite outgrowth. Cell 119:97-108.

Martinez I, Chakrabarti S, Hellevik T, Morehead J, Fowler K, Andrews NW (2000) Synaptotagmin VII regulates $\mathrm{Ca}(2+)$-dependent exocytosis of lysosomes in fibroblasts. J Cell Biol 148:1141-1149.

Martinez-Arca S, Alberts P, Zahraoui A, Louvard D, Galli T (2000) Role of tetanus neurotoxin insensitive vesicle-associated membrane protein (TI-VAMP) in vesicular transport mediating neurite outgrowth. J Cell Biol 149:889-900.

Martinez-Arca S, Coco S, Mainguy G, Schenk U, Alberts P, Bouille P, Mezzina M, Prochiantz A, Matteoli M, Louvard D, Galli T (2001) A common exocytotic mechanism mediates axonal and dendritic outgrowth. J Neurosci 21:3830-3838.

Moresco EM, Donaldson S, Williamson A, Koleske AJ (2005) Integrinmediated dendrite branch maintenance requires Abelson (Abl) family kinases. J Neurosci 25:6105-6118.

Overly CC, Hollenbeck PJ (1996) Dynamic organization of endocytic pathways in axons of cultured sympathetic neurons. J Neurosci 16:6056-6064.

Pandiella-Alonso A, Malgaroli A, Vicentini LM, Meldolesi J (1986) Early rise of cytosolic $\mathrm{Ca}^{2+}$ induced by NGF in PC12 and chromaffin cells. FEBS Lett 208:48-51.

Parton RG, Simons K, Dotti CG (1992) Axonal and dendritic endocytic pathways in cultured neurons. J Cell Biol 119:123-137.

Prochiantz A (1995) Neuronal polarity: giving neurons heads and tails. Neuron 15:743-746.

Rao SK, Huynh C, Proux-Gillardeaux V, Galli T, Andrews NW (2004) Identification of SNAREs involved in synaptotagmin VII-regulated lysosomal exocytosis. J Biol Chem 279:20471-20479.

Reddy A, Caler E, Andrews N (2001) Plasma membrane repair is mediated by $\mathrm{Ca}^{2+}$-regulated exocytosis of lysosomes. Cell 106:157-169.

Rehder V, Jensen JR, Kater SB (1992) The initial stages of neural regeneration are dependent upon intracellular calcium levels. Neuroscience 51:565-574.

Rodriguez A, Webster P, Ortego J, Andrews NW (1997) Lysosomes behave as $\mathrm{Ca}^{2+}$-regulated exocytic vesicles in fibroblasts and epithelial cells. J Cell Biol 137:93-104.

Ronn LC, Ralets I, Hartz BP, Bech M, Berezin A, Berezin V, Moller A, Bock E (2000) A simple procedure for quantification of neurite outgrowth based on stereological principles. J Neurosci Methods 100:25-32.

Rothman JE (1994) Mechanisms of intracellular protein transport. Nature 372:55-63. 
Roy D, Liston DR, Idone VJ, Di A, Nelson DJ, Pujol C, Bliska JB, Chakrabarti S, Andrews NW (2004) A process for controlling intracellular bacterial infections induced by membrane injury. Science 304:1515-1518.

Shoemaker SE, Sachs HH, Vaccariello SA, Zigmond RE (2005) A conditioning lesion enhances sympathetic neurite outgrowth. Exp Neurol 194:432-443.

Tolkovsky AM, Walker AE, Murrell RD, Suidan HS (1990) $\mathrm{Ca}^{2+}$ transients are not required as signals for long-term neurite outgrowth from cultured sympathetic neurons. J Cell Biol 110:1295-1306.

Tucker WC, Chapman ER (2002) Role of synaptotagmin in $\mathrm{Ca}^{2+}$-triggered exocytosis. Biochem J 366:1-13.

Wang P, Chicka MC, Bhalla A, Richards DA, Chapman ER (2005) Synaptotagmin VII is targeted to secretory organelles in PC12 cells, where it functions as a high-affinity calcium sensor. Mol Cell Biol 25:8693-8702.

Washbourne P, Thompson PM, Carta M, Costa ET, Mathews JR, Lopez-
Bendito G, Molnar Z, Becher MW, Valenzuela CF, Partridge LD, Wilson MC (2002) Genetic ablation of the t-SNARE SNAP-25 distinguishes mechanisms of neuroexocytosis. Nat Neurosci 5:19-26.

Weaver CM, Pinezich JD, Lindquist WB, Vazquez ME (2003) An algorithm for neurite outgrowth reconstruction. J Neurosci Methods 124:197-205.

Zakharenko S, Popov S (1998) Dynamics of axonal microtubules regulate the topology of new membrane insertion into the growing neurites. J Cell Biol 143:1077-1086.

Ziv NE, Spira ME (1997) Localized and transient elevations of intracellular $\mathrm{Ca}^{2+}$ induce the dedifferentiation of axonal segments into growth cones. J Neurosci 17:3568-3579.

Zoghbi HY (2003) Postnatal neurodevelopmental disorders: meeting at the synapse? Science 302:826-830. 